OPEN ACCESS

Edited by:

Suzie Chen,

Rutgers, The State University of New

Jersey, United States

Reviewed by:

Muhammad Nawaz,

University of Gothenburg, Sweden

Rosanna Botta,

National Institute of Health (ISS), Italy

*Correspondence:

Francesca Perut

francesca.perut@ior.it

Specialty section:

This article was submitted to

Cancer Molecular Targets and

Therapeutics,

a section of the journa

Frontiers in Oncology

Received: 27 September 2019 Accepted: 15 November 2019

Published: 03 December 2019

Citation:

Perut F, Roncuzzi L and Baldini N (2019) The Emerging Roles of

Extracellular Vesicles in

Osteosarcoma. Front. Oncol. 9:1342.

doi: 10.3389/fonc.2019.01342

\section{The Emerging Roles of Extracellular Vesicles in Osteosarcoma}

\author{
Francesca Perut ${ }^{1 *}$, Laura Roncuzzi ${ }^{1}$ and Nicola Baldini ${ }^{1,2}$ \\ ${ }^{1}$ Laboratory for Orthopedic Pathophysiology and Regenerative Medicine, IRCCS Istituto Ortopedico Rizzoli, Bologna, Italy, \\ ${ }^{2}$ Department of Biomedical and Neuromotor Sciences, University of Bologna, Bologna, Italy
}

Extracellular vesicles (EVs) are heterogeneous nanosized vesicles that are constitutively released by virtually all types of cells. They have been isolated in almost all body fluids. EVs cargo consists of various molecules (nucleic acids, proteins, lipids, and metabolites), that can be found on EVs surface and/or in their lumen. EVs structure confer stability and allow the transfer of their cargo to specific cell types over a distance. EVs play a critical role in intercellular communication in physiological and pathological settings. The broadening of knowledge on EVs improved our comprehension of cancer biology as far as tumor development, growth, metastasis, chemoresistance, and treatment are concerned. Increasing evidences suggest that EVs have a significant role in osteosarcoma (OS) development, progression, and metastatic process. The modulation of inflammatory communication pathways by EVs plays a critical role in OS and in other bone-related pathological conditions such as osteoarthritis and rheumatoid arthritis. In this review we describe the emerging data on the role of extracellular vesicles in osteosarcoma and discuss the effects and function of OS-derived EVs focusing on their future applicability in clinical practice.

Keywords: osteosarcoma, extracellular vesicles, multidrug resistance, liquid biopsy, microenvironment

\section{INTRODUCTION}

Extracellular vesicles are lipid bilayer nanovesicles containing nucleic acids (DNA, mRNA, and miRNA), proteins, metabolites and lipids (1). EVs were first described by Johnstone et al., that demonstrated EVs ability to transport transferrin receptor outside the cells during the maturation of reticulocyte (2). Recently, these nanovesicles have gained substantial attention as crucial factors in maintaining normal cellular and biological physiology. These vesicles are proposed to be tailormade specialized mini-maps of their cell of origin, and have peculiar functions in cell-to-cell communication (3). Extracellular vesicles are a class of nanovesicles including exosomes and microvesicles, that have been defined and sub-grouped on the basis of their size, biogenesis and composition. Exosomes are classically considered as $30-100 \mathrm{~nm}$, they are part of the endosomal compartment and are generated within large intracellular multivesicular bodies. They are released into the extracellular space upon fusion with the plasma membrane. Microvesicles range as $100-1,000 \mathrm{~nm}$ and are produced by direct budding from the plasmatic membrane (4).

The EVs lipid membrane protects its cargo from enzymatic degradation making them ideal carriers for local and long-distance transport (3). EVs have been identified in nearly all eukaryotic and prokaryotic cells and are secreted in physiological and pathological conditions $(5,6)$. They have been isolated from most body fluids including plasma, saliva and urine (7). Furthermore, extracellular vesicles are observed abundantly in tumor microenvironment where they play an 
important role in signaling pathways $(8,9)$. The presence of matrix metalloproteinases (MMPs) and MMP regulators in EVs showed their crucial role in extracellular matrix remodeling, that is involved not only in metastatic process but also in several bone-related conditions (10).

\section{OSTEOSARCOMA}

Osteosarcoma is the most common primary bone tumor and the more frequent pediatric solid cancer (11). To date, the standard treatment for osteosarcoma is based on neoadjuvant chemotherapy, surgery and post-operative chemotherapy. This aggressive treatment does not guarantee a favorable outcome, principally in patients with metastatic and/or recurrent disease $(12,13)$, thus new therapies are needed. Therefore, a major translational objective of osteosarcoma research is to identify new therapeutic markers and their clinical significance. In this review, we describe the emerging data on the role of EVs in osteosarcoma growth, metastasis, and chemoresistance (Figure 1), focusing on their future applicability in clinical practice.

\section{EFFECTS OF OS-DERIVED EVs ON CELLS OF TUMOR MICROENVIRONMENT}

The specific tumor-driving genetic alterations related to OS development are currently poorly understood. Although there is still no final consensus on the cell of origin for osteosarcoma, the regulation at epigenetic level may be an early event occurring in the transformation of mesenchymal stem cells (MSC) during OS development (14). Recently, Mannerström et al. examined the EV-mediated intercellular crosstalk of MSC and OS. The authors demonstrated that OS-EVs modulate the epigenetic status of MSC, through hypomethylation of long interspersed nuclear element 1. Furthermore, OS-derived EVs influenced the expression of matrix metallopeptidase 1, vascular endothelial growth factor A (VEGF-A), and intercellular adhesion molecule 1 which are related to bone microenvironment remodeling (15).

Tumor growth, progression, and metastatic process are supported by angiogenesis, that guarantees oxygen and nutrient supply to the OS expanding mass, and provides an escape route for tumor cells to enter the circulation and spread to distant organs (16). Different cells and mediators are involved in the angiogenic process. Recently, we highlighted the ability of OS derived-EVs to contribute to tumor angiogenesis. This pro-angiogenic activity is mediated by angiogenesis-related proteins (serpin-E1, serpin-F1, TIMP-1, thrombospondin-1, urokinase-type plasminogen activator (uPA), VEGF, pentraxin-3, PDGF-AA, angiopoietin-2, coagulation factor-III, CD26, CD105, endostatin, endothelin-1, and HB-EGF) and miRNAs (miR146a-5p, miR-10b-5p, miR-143-3p, miR-382-5p, miR-150-5p, miR-125b-5p, miR-27a-3p, miR-145-5p, miR-26a-5p, miR-93$5 p$, miR-21-5p, miR-92a-3p, and miR-106a-5p) that have been identified in OS derived-EVs cargo (17). Moreover, Raimondi et al. showed that OS derived-EVs stimulated endothelial cells to express and secrete elevated levels of the pro-angiogenic factor VEGF, and interleukins (IL-6 and IL-8) (18). The role of tumor-derived EVs cargo in stimulation of angiogenesis is wellrecognized in other tumors such as multiple myeloma, glioma, renal cell carcinoma, pancreatic, colorectal, prostate, and lung cancer (19). Thus, angiogenic EV cargo can now be reasonably considered a common aggressive trait of cancer derived-EVs.

Osteosarcoma is generally considered a tumor of osteoblastic lineage, and it has been demonstrated that osteoclasts have a crucial role in OS aggressiveness and poor response to chemotherapy $(20,21)$. Osteoclast formation and bone resorption are stimulated by osteoclast-inducing factors secreted by OS cells themselves, and, additionally, by the proosteoclastogenic cargo of OS-derived EVs $(18,22)$. In particular, the biochemical characterization of OS-derived EVs identified a profile of bioactive pro-osteoclastic factors including matrix metalloproteinase-1 and 13, transforming growth factor $\beta$ (TGF- $\beta$ ), CD- 9 and receptor activator of nuclear factor kappa- $\beta$ ligand (RANKL) (22). In addition, Raimondi et al. identified a pro-osteoclastic miRNA cargo in OS-derived EVs. The authors demonstrated that EVs contain miR-148a-3p and miR-21-5p, known for their involvement in the tumor microenvironment establishment (18). The functional role of miR-148a-3p in bone homeostasis, osteoclastogenesis and bone metabolism in vivo has been previously described (23). Additionally, miR-21-5p has been found highly expressed in osteoclast precursors, and upregulated during RANKL-induced osteoclastogenesis (24). These studies suggest a specific role of the pro-osteoclastogenic cargo of EVs in the alteration of bone remodeling homeostasis in OS bone microenvironment.

The contribution of EVs in tumor progression and metastatic process may be exerted through both local and distant intercellular communication. Macklin et al. demonstrated a role of EVs as mediators in the transfer of migratory and invasive characteristics from OS subclones with highly metastatic traits to poor metastatic cells (25). The hypothesis that a local interclonal cooperation through EV production and transfer favor the metastatic progression of OS, and can determine organotropic metastasis by inducing a pre-metastatic niche, has already been previously demonstrated for human breast and pancreatic cancer (26).

OS derived-EVs may furthermore contribute to metastatic process by prompting MSC to acquire a pro-tumorigenic and pro-metastatic phenotype. Indeed, OS derived-EVs cells selectively incorporate a membrane-associated form of TGF- $\beta$, which induces the pro-inflammatory IL- 6 production by MSC. MSC-derived IL-6 increases tumor growth and metastasis formation in mice bearing osteosarcoma $(8,27)$. Moreover, OS cells secreted both the soluble form of uPA and uPA-containing exosomes. Interestingly, the autocrine and paracrine activation of the UPA/uPAR axis has been related to the conversion of OS cells to a metastatic phenotype (28).

The interaction of OS cells with the surrounding immune cells has been explored to support immunotherapy approaches for OS and their potential use as adjuvant therapies (29). The analysis of the proteomic composition of OS-derived EVs in a canine osteosarcoma model identified immunosuppressive proteins with immunomodulatory effects on T cells. In particular, authors demonstrated a diminished activation and proliferation 


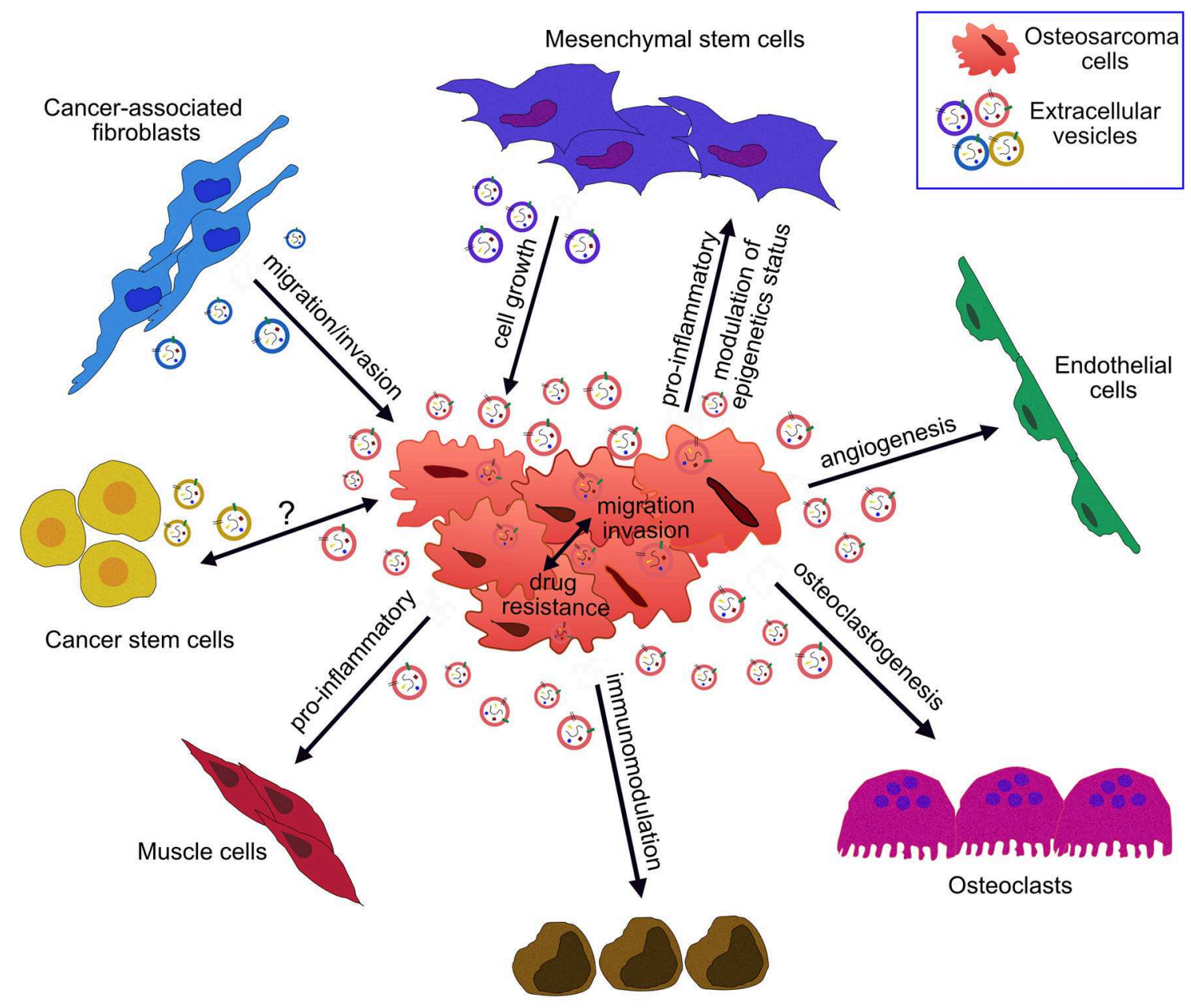

T cells

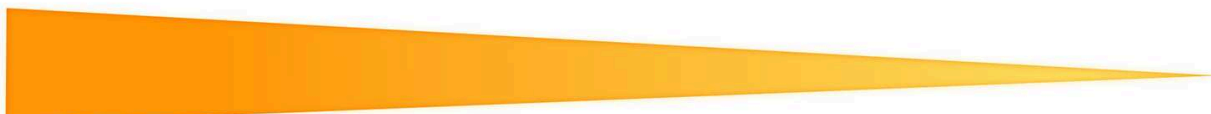

Microenvironment changes (i.e. hypoxia, acidic $\mathrm{pH}$, stress,...)

EVs amount and cargo

FIGURE 1 | Role of extracellular vesicles in the communication between osteosarcoma cells and the tumor microenvironment. Osteosarcoma cells interact with the surrounding cells through secretion and up-take of extracellular vesicles (EVs). EVs cargo consists of nucleic acids, proteins, lipids, and metabolites. EVs have been found to play roles in a multitude of pathways involved in tumor growth, progression and metastatic process. EV-mediated crosstalk occurs through the trafficking of vesicle-associated components to endothelial cells, osteoclasts, T cells, muscle cells, cancer stem cells, cancer-associated fibroblasts, mesenchymal stem cells, and osteosarcoma cells. OS-derived EVs influence angiogenesis, osteoclastogenesis, immunomodulation, drug resistance, epigenetics status, invasion, and migration processes. EVs derived from cells of OS microenvironment affect OS growth, migration, and invasion. Peculiar microenvironment tumor conditions (acidic pH, hypoxia and stress) affect EVs secretion and features.

of CD4+ and CD8+ T cells (30). Cancer derived-EVs have been linked with several mechanisms that support tumor development through immune escape $(31,32)$. In particular, the activation of the programmed death ligand 1 (PD-L1) pathway is used by cancer cells in the process of immune surveillance evasion, and the overexpression of PD-L1 has been associated with increased metastasis in OS (33). Interestingly, Chen et al. showed that metastatic melanoma released EVs carrying PD-L1 that can be used to predict the effect of anti-PD-1 therapy (32).

OS-derived EVs may also play a role in sarcoma-associated cachexia, secondary to aggressive surgical treatment of OS. Interestingly, $\mathrm{Mu}$ et al. have related inflammation and the 
crosstalk between NF-kB and Notch signaling to skeletal muscle atrophy seen in cancer cachexia, and demonstrated that EVs derived from OS murine cells may transfer Notch-activating signals to muscle cells (34).

So far, there are no data available on EVs derived from OS cancer stem cells (CSC), that represent a sub-type of tumor cells with well-known roles in tumor propagation, therapy resistance, recurrence, and metastasis (35). In prostate cancer a different miRNA pattern was found in EV cargo derived from tumor bulk and CSC, thus unveiling additional potential biomarkers and therapeutic targets (36). In this direction, we may assume that future investigations of EV cargo derived from OS CSC will enrich the panel of EV-derived biomarkers.

To summarize, different specific activities of OS-derived EVs on tumor microenvironment cells have been demonstrated. However, according to Jerez et al. the enrichment of EV proteins and miRNA derived from different OS cell lines is heterogeneous $(37,38)$. This has to be carefully considered when general conclusion on OS behavior are based on a specific miRNAs or protein identified in EV cargo derived by a specific cell line. In order to substantiate the findings, use of additional cell lines and, especially possibly, primary OS cells should be considered in future experiments.

\section{FUNCTIONS OF EVS DERIVED FROM CELLS OF OS MICROENVIRONMENT}

MSC and cancer-associated fibroblasts (CAFs) are known to support tumor progression and chemoresistance, through paracrine cross-feeding and vesicles secretion (39). Thus, a growing interest has been devoted to explore the activity of EVs derived from MSC or CAF on tumor cells. The transfer of EVs derived miRNA represents an additional level of intercellular communication between stroma and cancer cells. Recently, Wang et al. demonstrated that miR1228 encapsulated in CAF-derived EVs promotes OS cells migration and invasion by downregulating suppressor cancer cell invasion mRNA expression (40). Recent findings suggest that the contribution of MSC-derived EVs to OS growth is mediated by the activation of Hedgehog signaling pathway (41). Moreover, Lin et al. demonstrated that MSC-derived microvesicles support U2OS cell growth under hypoxia, and that this activity was partially related with the PI $3 \mathrm{~K} / \mathrm{AKT}$ and HIF- $1 \alpha$ pathways (42).

However, MSC and CAF are not the unique cellular component of osteosarcoma microenvironment. Indeed, osteoblasts, osteoclasts, endothelial, and immune cells coexist with cancer cells and participate in their growth and survival. Moreover, their ability to secrete EVs has already been demonstrated in other pathophysiological models (43-45). Further investigation into how EVs derived from microenvironment cells may act on OS cells will help to elucidate new factors and mechanisms involved in cell communication within tumor microenvironment, and to discover new targets and/or biomarkers.

\section{INFLUENCE OF MICROENVIRONMENT ON EVs SECRETION AND FUNCTION}

Several microenvironment parameters (acidosis, hypoxia, and elevated interstitial fluid pressure) influence tumor cell viability, proliferation, motility and metabolism, and are able to lead to more aggressive behavior of OS cells (46). Malignant lesions of mesenchymal tumors appear to be quite acidic (47), and it has been demonstrated that extracellular acidosis contributes to OS behavior, chemoresistance, and response to therapy (4850). Interestingly, Logozzi et al. demonstrated that an acid extracellular $\mathrm{pH}$ (6.5) induced a significant increase in EV release, while buffering the medium reduced the EV release in prostate, melanoma, osteosarcoma, breast, adenocarcinoma, and colorectal carcinoma cells (51). According to this, we recently demonstrated an increased amount of EV protein mass secreted by OS cells grown in acidic compared to neutral $\mathrm{pH}$, and a higher pro-angiogenic activity of acidic OS-derived EVs on in vivo assay (17). Different hypothesis have been discussed to determine how EVs cargo is transferred from cell to cell, without reaching a conclusive and accepted theory (52). Despite this, Parolini et al. demonstrated an increased fusion efficiency of EVs secreted at low extracellular $\mathrm{pH}$ by melanoma cells, possibly due to the modified lipid composition of EVs detected at acidic $\mathrm{pH}$ (53). The pharmacological handling of the extracellular and intracellular $\mathrm{pH}$ of cancers, that has been considered as a potential additional treatment in tumor therapy (54), may thus also interfere with EVs release and fusion efficiency.

Stress conditions are likewise able to modify the behavior of cells of tumor microenvironment. It is well-known that hypoxia, poor nutrient conditions, and mechanical stress influence MSC secretome (55-57). In this context, it is not surprising that EVs isolated from serum deprived MSC carries tumor supportive miRNA and lncRNA, and increase OS survival and resistance to apoptosis $(58,59)$.

As microenvironment parameters influence EVs release and cargo, an emerging concept is to produce more sophisticated in vitro models to better resemble in vivo cell environment when studying EVs, as it is already a well-recognized approach in drug discovery (60). According to this, Villasante et al. pointed out that 3-dimensionality and stiffness of a tumor matrix can determine the size and cargo of EVs released by Ewing's sarcoma cells. These authors suggested to study EVs in $3 \mathrm{D}$ rather than in $2 \mathrm{D}$ setting to better mimic the native structure of the tumor (61).

\section{ROLE OF OS-DERIVED EVs IN ANTI-CANCER DRUG RESISTANCE}

Multidrug resistance (MDR), intrinsic or acquired, remains a major obstacle to successful osteosarcoma treatment and contributes to poor clinical outcome (62). Recent studies support EVs as playing a key role in OS drug-resistance (63-66). We demonstrated that the MDR phenotype can be induced in OS cells through MDR OS-derived EVs. These nanovesicles are able to decrease OS cell sensitivity to doxorubicin by the transfer of functional MDR-1 mRNA, 
TABLE 1 | Biomarkers identified in circulating EVs in osteosarcoma.

\begin{tabular}{|c|c|c|c|}
\hline Biomarker & Sample Type & Number of patients analyzed & References \\
\hline EV-associated TGF $\beta$ & Human serum & $\begin{array}{l}n=10 \text { OS patients } \\
n=10 \text { healthy donors }\end{array}$ & $(24)$ \\
\hline $\begin{array}{l}\text { EV-associated SERPING1, } \\
\text { HEL-S-71p, HBB, KRT10, HEL180, } \\
\text { TIH1, IGLC7, DC33, and } \\
\text { characterized protein }\end{array}$ & Canine serum & $\begin{array}{l}n=8 \text { OS group } \\
n=5 \text { healthy dogs with } \\
\text { traumatic bone fractures } \\
n=5 \text { healthy, } \\
\text { size-matched controls }\end{array}$ & $(68)$ \\
\hline $\begin{array}{l}\text { EV-associated SERPIND1 and class } \\
\text { III MHC }\end{array}$ & Canine serum & $\begin{array}{l}n=5 \text { OS diagnosis } \\
n=5,2 \text { weeks after amputation } \\
n=5 \text { onset of lung metastases }\end{array}$ & $(68)$ \\
\hline $\begin{array}{l}\text { Serum exosomal miRNAs: } \\
\text { miR-124, miR133a, miR-9, } \\
\text { miR199a-3p, miR-385, miR-135b, } \\
\text { miR-148a, miR-27a } \\
\text { Serum exosomal mRNAs: } \\
\text { Annexin2, Smad2, MTAP, CIP4, } \\
\text { PEDF, WWOX, Cdc5L, P27 }\end{array}$ & Human serum & $\begin{array}{l}n=48 \text { OS patients with poor } \\
\text { chemotherapeutic } \\
n=45 \text { OS patients with good } \\
\text { chemotherapeutic response } \\
n=51 \text { healthy donors }\end{array}$ & $(69)$ \\
\hline EVRNA carries aberrant gene fusions & Human plasma & $\begin{array}{l}n=3 \text { OS presurgery patients } \\
n=3 \text { OS metastatic patients }\end{array}$ & $(70)$ \\
\hline Mutations of RNA in circulating EVs & Human blood & $\begin{array}{l}n=40 \text { metastatic and } \\
\text { non-metastatic patients } \\
\text { Recruiting is still ongoing. }\end{array}$ & $\begin{array}{l}\text { ClinicalTrials.gov } \\
\text { Identifier: NCT03108677 }\end{array}$ \\
\hline
\end{tabular}

and its product P-glycoprotein, inducing MDR phenotype to OS doxorubicin-sensitive cells (65). Recently, pre-clinical and clinical data linked EVs to MDR also in hematological malignancies, glioblastoma, neuroblastoma, melanoma, breast, prostate, lung, ovarian, colorectal, gastric, pancreatic, and kidney cancer $(66,67)$.

Moreover, EVs can mediate MDR through the transfer of specific bioactive molecules including, prosurvival/apoptosis related-factors, and non-coding RNAs (68). Much attention has focused on the miRNAs identified in EVs cargo due to their capacity to interfere in gene regulation and subsequently to be involved in a variety of drug resistance pathways and mechanisms (69). Therefore, the EV and its molecular cargo can be viewed as a fundamental mediator of cancer drug resistance.

\section{EVs AS BIOMARKERS IN OS}

Liquid biopsy strategies are now being explored to discover and validate new and more efficient and/or complementary approaches to improve OS diagnosis, management and treatment (70). Liquid biopsies can be profitably used to assess molecular heterogeneity of OS tumors, and to provide dynamic tumor information. In this context, EVs represent a promising target as they can be easily non-invasively isolated from accessible body fluids including blood, urine and saliva. Furthermore, EV cargo is protected from degradation inside a membranous structure, that provides stability and allows prolonged periods of storage of EVs before analysis, making their clinical use feasible (71). EVs contains nucleic acids, proteins, lipids and metabolites that can be identified, characterized and thus used as biomarkers. As far as EV-associated proteins are concerned, circulating levels of EV-associated TGF- $\beta$ have been found to be increased in osteosarcoma patients, when compared to healthy control subjects (27). A proteomic investigation of circulating EVs in canine serum samples identified EV related proteins useful to distinguish serum of osteosarcoma from serum of healthy or fractured dogs (72). Furthermore, Brady et al. identified two proteins associated with EVs (serpinD1 and MHC class III-complement C6) which allow to discriminate serum derived from different disease stages of OS (72).

The presence of a specific collection of RNAs in EVs cargo may also serve as new or supplementary biomarker in OS diagnosis and progression. Xu et al. showed dysregulated levels of several miRNAs and mRNAs in EVs isolated from serum of OS patients with a poor chemotherapeutic response when compared with good responders (73). Moreover, Bao et al. demonstrated in a pilot study, an increased tumor mutation burden in RNA isolated from metastatic EVs plasma samples compared to non-metastatic ones (74). A prospective observational study to reveal the roles of circulating EVs RNA in lung metastases of primary high-grade osteosarcoma was launched in 2017 and recruiting is still ongoing (ClinicalTrials.gov: Identifier: NCT03108677).

In this review different EVs cargo components have been described as potential biomarkers in OS patients. In Table 1 biomarkers identified in circulating EVs in osteosarcoma are reported. The number of patients and source of EVs (plasma, serum, or blood) were described. These studies were carried 
out in small cohorts of patients utilizing different methods to isolate EVs. According to Ayers et al. several parameters and challenges will have to be considered before a diagnostic clinical application of EVs can become a solid reality (75). The major concern of all studies using EVs are focused on standardization and improvement of methods to isolate EVs and, if appropriate, to distinguish EV subpopulations. It has to be pointed out that also a standardization of pre-analytical variables is also required to ensure that the quantity and characteristics of EVs reported can be reliably evaluated. The possibility to merge data coming from different laboratories would be simplified by following shared protocols and guidelines. In this respect the International Society of Extracellular Vesicles supported several initiatives to favor method homogenization, such as the EV Transparent Reporting and Centralizing Knowledge (76), the Minimal Information for Studies of EVs (4) and the Clinical Wrap-Up session at ISEV2018 (77). As far as EVs isolation methods are concerned, microfluidic miniaturized systems have recently emerged as promising technology to address both isolation and analysis of EVs in clinical settings, where a small amount of samples are available and rare molecular targets have to be detected $(78,79)$.

\section{CONCLUSIONS}

Osteosarcoma is a complex system in which cancer cells, cancer stem cells, mesenchymal cells, immune cells, fibroblasts and endothelial cells coexist and communicate. Recently acquired

\section{REFERENCES}

1. Pathan M, Fonseka P, Chitti SV, Kang T, Sanwlani R, Van Deun J, et al. Vesiclepedia 2019: a compendium of RNA, proteins, lipids and metabolites in extracellular vesicles. Nucleic Acids Res. (2019) 47:D516-9. doi: 10.1093/nar/gky1029

2. Johnstone RM, Adam M, Hammond JR, Orr L, Turbide C. Vesicle formation during reticulocyte maturation. Association of plasma membrane activities with released vesicles (exosomes). J Biol Chem. (1987) 262:9412-20.

3. Lopez-Verrilli MA, Court FA. Exosomes: mediators of communication in eukaryotes. Biol Res. (2013) 46:5-11. doi: 10.4067/S0716-97602013000100001

4. Théry C, Witwer KW, Aikawa E, Alcaraz MJ, Anderson JD, Andriantsitohaina $\mathrm{R}$, et al. Minimal information for studies of extracellular vesicles 2018 (MISEV2018): a position statement of the International Society for Extracellular Vesicles and update of the MISEV2014 guidelines. J Extracell Vesicles. (2018) 8:1535750. doi: 10.1080/20013078.2018.1535750

5. Deatherage BL, Cookson BT. Membrane vesicle release in bacteria, eukaryotes, and archaea: a conserved yet underappreciated aspect of microbial life. Infect Immun. (2012) 80:1948-57. doi: 10.1128/IAI.06014-11

6. Raposo G, Stoorvogel W. Extracellular vesicles: exosomes, microvesicles, and friends. J Cell Biol. (2013) 200:373-83. doi: 10.1083/jcb.2012 11138

7. Colombo M, Raposo G, Théry C. Biogenesis, secretion, and intercellular interactions of exosomes and other extracellular vesicles. Annu Rev Cell Dev Biol. (2014) 30:255-89. doi: 10.1146/annurev-cellbio-101512-122326

8. Lan M, Zhu XP, Cao ZY, Liu JM, Lin Q, Liu ZL. Extracellular vesiclesmediated signaling in the osteosarcoma microenvironment: Roles and potential therapeutic targets. J Bone Oncol. (2018) 12:101-4. doi: 10.1016/j.jbo.2018.07.010 knowledge indicates that the interactions among these cells are also mediated by extracellular vesicles. The transfer of tumor-supportive traits from osteosarcoma cells to donor cells contribute to cancer growth and metastatic process. On the other hand, EVs derived from microenvironment cells are able to influence tumor growth and migration. Additionally, specific tumor microenvironment features (stress, hypoxia, acidic $\mathrm{pH}$ ) interfere with EV secretion and cargo. Overall, data from literature suggest multiple functions of EVs in osteosarcoma, paving the way to discover new therapeutic targets and to design innovative diagnostic assays. Future efforts must focus on technological advances in EV purification and characterization to improve EV detection and cargo evaluation in clinical setting. Moreover, one additional challenge for the future will be to associate new functions to subclasses of EVs to identify peculiar pathways involving EVs, and changes in their cargo related to OS stage or response to therapy.

\section{AUTHOR CONTRIBUTIONS}

All authors listed have made a substantial, direct and intellectual contribution to the work, and approved it for publication.

\section{FUNDING}

Support was provided by grant funding to NB (The Italian Ministry of the Health, Financial Support for Scientific Research (5 per 1000 2017).
9. Becker A, Thakur BK, Weiss JM, Kim HS, Peinado H, Lyden D. Extracellular vesicles in cancer: cell-to-cell mediators of metastasis. Cancer Cell. (2016) 30:836-48. doi: 10.1016/j.ccell.2016.10.009

10. Nawaz M, Shah N, Zanetti BR, Maugeri M, Silvestre RN, Fatima F, et al. Extracellular vesicles and matrix remodeling enzymes: the emerging roles in extracellular matrix remodeling, progression of diseases and tissue repair. Cells. (2018) 7:167. doi: 10.3390/cells7100167

11. Lindsey BA, Markel JE, Kleinerman ES. Osteosarcoma overview. Rheumatol Ther. (2017) 4:25-43. doi: 10.1007/s40744-016-0050-2

12. Miller BJ, Cram P, Lynch CF, Buckwalter JA. Risk factors for metastatic disease at presentation with osteosarcoma: an analysis of the SEER database. J Bone Joint Surg Am. (2013) 95:e89. doi: 10.2106/JBJS.L.01189

13. Biazzo A, De Paolis M. Multidisciplinary approach to osteosarcoma. Acta Orthop Belg. (2016) 82:690-8.

14. Yang Y, Yang R, Roth M, Piperdi S, Zhang W, Dorfman H, et al. Genetically transforming human osteoblasts to sarcoma: development of an osteosarcoma model. Genes Cancer. (2017) 8:484-94. doi: 10.18632/genesandcancer.133

15. Mannerström B, Kornilov R, Abu-Shahba AG, Chowdhury IM, Sinha S, Seppänen-Kaijansinkko R, et al. Epigenetic alterations in mesenchymal stem cells by osteosarcoma-derived extracellular vesicles. Epigenetics. (2019) 14:352-64. doi: 10.1080/15592294.2019.1585177

16. Li YS, Liu Q, Tian J, He HB, Luo W. Angiogenesis process in osteosarcoma: an updated perspective of pathophysiology and therapeutics. Am J Med Sci. (2019) 357:280-88. doi: 10.1016/j.amjms.2018.12.004

17. Perut F, Roncuzzi L, Zini N, Massa A, Baldini N. Extracellular nanovesicles secreted by human osteosarcoma cells promote angiogenesis. Cancers. (2019) 11:779. doi: 10.3390/cancers11060779

18. Raimondi L, De Luca A, Gallo A, Costa V, Russelli G, Cuscino N, et al. Osteosarcoma cell-derived exosomes affect tumor microenvironment 
by specific packaging of microRNAs. Carcinogenesis. (2019) 1-12. doi: 10.1093/carcin/bgz130

19. Aslan C, Maralbashi S, Salari F, Kahroba H, Sigaroodi F, Kazemi T, et al. Tumor-derived exosomes: implication in angiogenesis and antiangiogenesis cancer therapy. J Cell Physiol. (2019) 234:16885-903. doi: 10.1002/jcp.28374

20. Avnet S, Longhi A, Salerno M, Halleen JM, Perut F, Granchi D, et al. Increased osteoclast activity is associated with aggressiveness of osteosarcoma. Int $J$ Oncol. (2008) 33:1231-38 doi: 10.1016/j.bone.2007.12.174

21. Bago-Horvath Z, Schmid K, Rössler F, Nagy-Bojarszky K, Funovics P, Sulzbacher I. Impact of RANK signalling on survival and chemotherapy response in osteosarcoma. Pathology. (2014) 46:411-15. doi: 10.1097/PAT.0000000000000116

22. Garimella R, Washington L, Isaacson J, Vallejo J, Spence M, Tawfik O, et al. Extracellular membrane vesicles derived from 143B osteosarcoma cells contain pro-osteoclastogenic cargo: a novel communication mechanism in osteosarcoma bone microenvironment. Transl Oncol. (2014) 7:331-40. doi: 10.1016/j.tranon.2014.04.011

23. Cheng $\mathrm{P}$, Chen $\mathrm{C}, \mathrm{He} \mathrm{HB}, \mathrm{Hu} \mathrm{R}$, Zhou HD, Xie H, et al. miR148a regulates osteoclastogenesis by targeting V-maf musculoaponeurotic fibrosarcoma oncogene homolog B. J Bone Miner Res. (2013) 28:1180-90. doi: $10.1002 / j b m r .1845$

24. Ji X, Chen X, Yu X. MicroRNAs in osteoclastogenesis and function: potential therapeutic targets for osteoporosis. Int J Mol Sci. (2016) 17:349. doi: $10.3390 /$ ijms 17030349

25. Macklin R, Wang H, Loo D, Martin S, Cumming A, Cai N, et al. Extracellular vesicles secreted by highly metastatic clonal variants of osteosarcoma preferentially localize to the lungs and induce metastatic behaviour in poorly metastatic clones. Oncotarget. (2016) 7:43570-87. doi: 10.18632/oncotarget.9781

26. Hoshino A, Costa-Silva B, Shen TL, Rodrigues G, Hashimoto A, Tešić Mark $\mathrm{M}$, et al. Tumour exosome integrins determine organotropic metastasis. Nature. (2015) 527:329-35. doi: 10.1038/nature15756

27. Baglio SR, Lagerweij T, Pérez-Lanzón M, Ho XD, Léveillé N, Melo SA, et al. Blocking tumor-educated MSC paracrine activity halts osteosarcoma progression. Clin Cancer Res. (2017) 23:3721-33. doi: 10.1158/1078-0432.CCR-16-2726

28. Endo-Munoz L, Cai N, Cumming A, Macklin R, Merida de Long L, Topkas E, et al. Progression of osteosarcoma from a non-metastatic to a metastatic phenotype is causally associated with activation of an autocrine and paracrine uPA axis. PLoS ONE. (2015) 10:e0133592. doi: 10.1371/journal.pone.0133 592

29. Miwa S, Shirai T, Yamamoto N, Hayashi K, Takeuchi A, Igarashi K, et al. Current and emerging targets in immunotherapy for osteosarcoma. J Oncol. (2019) 2019:7035045. doi: 10.1155/2019/7035045

30. Troyer RM, Ruby CE, Goodall CP, Yang L, Maier CS, Albarqi HA, et al. Exosomes from osteosarcoma and normal osteoblast differ in proteomic cargo and immunomodulatory effects on T cells. Exp Cell Res. (2017) 358:369-76. doi: 10.1016/j.yexcr.2017.07.011

31. Clayton A, Mitchell JP, Court J, Mason MD, Tabi Z. Human tumor-derived exosomes selectively impair lymphocyte responses to interleukin-2. Cancer Res. (2007) 67:7458-66 doi: 10.1158/0008-5472.CAN-06-3456

32. Chen G, Huang AC, Zhang W, Zhang G, Wu M, Xu W, et al. Exosomal PD-L1 contributes to immunosuppression and is associated with anti-PD-1 response. Nature. (2018) 560:382-86. doi: 10.1038/s41586-018-0392-8

33. Huang X, Zhang W, Zhang Z, Shi D, Wu F, Zhong B, et al. Prognostic value of programmed cell death 1 ligand-1 (PD-L1) or PD-1 expression in patients with osteosarcoma: a meta-analysis. J Cancer. (2018) 9:2525-531. doi: 10.7150/jca.25011

34. Mu X, Agarwal R, March D, Rothenberg A, Voigt C, Tebbets J, et al. Notch signaling mediates skeletal muscle atrophy in cancer cachexia caused by osteosarcoma. Sarcoma. (2016) 2016:3758162. doi: 10.1155/2016/3758162

35. Brown HK, Tellez-Gabriel M, Heymann D. Cancer stem cells in osteosarcoma. Cancer Lett. (2017) 386:189-95. doi: 10.1016/j.canlet.2016.11.019

36. Sánchez CA, Andahur EI, Valenzuela R, Castellón EA, Fullá JA, Ramos $\mathrm{CG}$, et al. Exosomes from bulk and stem cells from human prostate cancer have a differential microRNA content that contributes cooperatively over local and pre-metastatic niche. Oncotarget. (2016) 7:3993-4008. doi: 10.18632 /oncotarget. 6540
37. Jerez S, Araya H, Thaler R, Charlesworth MC, López-Solís R, Kalergis AM, et al. Proteomic analysis of exosomes and exosome-free conditioned media from human osteosarcoma cell lines reveals secretion of proteins related to tumor progression. J Cell Biochem. (2017) 118:351-60. doi: 10.1002/jcb.25642

38. Jerez $\mathrm{S}$, Araya $\mathrm{H}$, Hevia $\mathrm{D}$, Irarrázaval $\mathrm{CE}$, Thaler $\mathrm{R}$, van Wijnen $\mathrm{AJ}$, et al. Extracellular vesicles from osteosarcoma cell lines contain miRNAs associated with cell adhesion and apoptosis. Gene. (2019) 710:246-57. doi: 10.1016/j.gene.2019.06.005

39. Cortini M, Avnet S, Baldini N. Mesenchymal stroma: role in osteosarcoma progression. Cancer Lett. (2017) 405:90-9. doi: 10.1016/j.canlet.2017.07.024

40. Wang JW, Wu XF, Gu XJ, Jiang XH. Exosomal miR-1228 from cancer-associated fibroblasts promotes cell migration and invasion of osteosarcoma by directly targeting SCAI. Oncol Res. (2019) 27:979-86. doi: $10.3727 / 096504018 X 15336368805108$

41. Qi J, Zhou Y, Jiao Z, Wang X, Zhao Y, Li Y, et al. Exosomes derived from human bone marrow mesenchymal stem cells promote tumor growth through hedgehog signaling pathway. Cell Physiol Biochem. (2017) 42:224254. doi: 10.1159/000479998

42. Lin S, Zhu B, Huang G, Zeng Q, Wang C. Microvesicles derived from human bone marrow mesenchymal stem cells promote U2OS cell growth under hypoxia: the role of PI3K/AKT and HIF-1 $\alpha$. Hum Cell. (2019) 32:64-74. doi: 10.1007/s13577-018-0224-Z

43. Sun W, Zhao C, Li Y, Wang L, Nie G, Peng J, et al. Osteoclast-derived microRNA-containing exosomes selectively inhibit osteoblast activity. Cell Discov. (2016) 2:16015. doi: 10.1038/celldisc.2016.15

44. Jansen F, Li Q, Pfeifer A, Werner N. Endothelial- and immune cell-derived extracellular vesicles in the regulation of cardiovascular health and disease. JACC Basic Transl Sci. (2017) 2:790-807. doi: 10.1016/j.jacbts.2017.08.004

45. Tang H, He Y, Li L, Mao W, Chen X, Ni H, et al. Exosomal MMP2 derived from mature osteoblasts promotes angiogenesis of endothelial cells via VEGF/Erk1/2 signaling pathway. Exp Cell Res. (2019) 29:111541. doi: 10.1016/j.yexcr.2019.111541

46. Matsubara T, Diresta GR, Kakunaga S, Li D, Healey JH. Additive influence of extracellular ph, oxygen tension, and pressure on invasiveness and survival of human osteosarcoma cells. Front Oncol. (2013) 3:199. doi: $10.3389 /$ fonc. 2013.00199

47. Matsubara T, Kusuzaki K, Matsumine A, Shintani K, Satonaka H, Uchida A. Acridine orange used for photodynamic therapy accumulates in malignant musculoskeletal tumors depending on $\mathrm{pH}$ gradient. Anticancer Res. (2006) 26:187-93.

48. Ferrari S, Perut F, Fagioli F, Brach Del Prever A, Meazza C, Parafioriti A, et al. Proton pump inhibitor chemosensitization in human osteosarcoma: from the bench to the patients' bed. J Transl Med. (2013) 11:268. doi: 10.1186/1479-5876-11-268

49. Avnet S, Lemma S, Cortini M, Pellegrini P, Perut F, Zini N, et al. Altered $\mathrm{pH}$ gradient at the plasma membrane of osteosarcoma cells is a key mechanism of drug resistance. Oncotarget. (2016) 7:63408-423. doi: 10.18632/oncotarget.11503

50. Chano T, Avnet S, Kusuzaki K, Bonuccelli G, Sonveaux P, Rotili D, et al. Tumour-specific metabolic adaptation to acidosis is coupled to epigenetic stability in osteosarcoma cells. Am J Cancer Res. (2016) 6:859-75.

51. Logozzi M, Mizzoni D, Angelini DF, Di Raimo R, Falchi M, Battistini $\mathrm{L}$, et al. Microenvironmental $\mathrm{pH}$ and exosome levels interplay in human cancer cell lines of different histotypes. Cancers. (2018) 10:370. doi: $10.3390 /$ cancers 10100370

52. Mathieu M, Martin-Jaular L, Lavieu G, Théry C. Specificities of secretion and uptake of exosomes and other extracellular vesicles for cell-to-cell communication. Nat Cell Biol. (2019) 21:9-17. doi: 10.1038/s41556-018-0250-9

53. Parolini I, Federici C, Raggi C, Lugini L, Palleschi S, De Milito A, et al. Microenvironmental $\mathrm{pH}$ is a key factor for exosome traffic in tumour cells. J Biol Chem. (2009) 284:34211-22. doi: 10.1074/jbc.M109.041152

54. McCarty MF, Whitaker J. Manipulating tumor acidification as a cancer treatment strategy. Altern Med Rev. (2010) 15:264-72.

55. Swamynathan P, Venugopal P, Kannan S, Thej C, Kolkundar U, Bhagwat S, et al. Are serum-free and xeno-free culture conditions ideal for large scale clinical grade expansion of Wharton's jelly derived mesenchymal stem cells? A comparative study. Stem Cell Res Ther. (2014) 5:88. doi: 10.1186/scrt477 
56. De Lisio M, Jensen T, Sukiennik RA, Huntsman HD, Boppart MD. Substrate and strain alter the muscle-derived mesenchymal stem cell secretome to promote myogenesis. Stem Cell Res Ther. (2014) 5:74. doi: 10.1186/scrt463

57. Teixeira FG, Panchalingam KM, Anjo SI, Manadas B, Pereira R, Sousa N, et al. Do hypoxia/normoxia culturing conditions change the neuroregulatory profile of Wharton Jelly mesenchymal stem cell secretome? Stem Cell Res Ther. (2015) 6:133. doi: 10.1186/s13287-015-0124-Z

58. Vallabhaneni KC, Penfornis P, Dhule S, Guillonneau F, Adams KV, Mo YY, et al. Extracellular vesicles from bone marrow mesenchymal stem/stromal cells transport tumor regulatory microRNA, proteins, and metabolites. Oncotarget. (2015) 6:4953-67. doi: 10.18632/oncotarget.3211

59. Vallabhaneni KC, Hassler MY, Abraham A, Whitt J, Mo YY, Atfi A, et al. Mesenchymal stem/stromal cells under stress increase osteosarcoma migration and apoptosis resistance via extracellular vesicle mediated communication. PLoS ONE. (2016) 11:e0166027. doi: 10.1371/journal.pone.0166027

60. Langhans SA. Three-dimensional in vitro cell culture models in drug discovery and drug repositioning. Front Pharmacol. (2018) 9:6. doi: 10.3389/fphar.2018.00006

61. Villasante A, Marturano-Kruik A, Ambati SR, Liu Z, Godier-Furnemont A, Parsa H, et al. Recapitulating the size and cargo of tumor exosomes in a tissueengineered model. Theranostics. (2016) 6:1119-30. doi: 10.7150/thno.13944

62. Li S, Sun W, Wang H, Zuo D, Hua Y, Cai Z. Research progress on the multidrug resistance mechanisms of osteosarcoma chemotherapy and reversal. Tumor Biol. (2015) 36: 1329-38. doi: 10.1007/s13277-015-3181-0

63. Chen Z, Shi T, Zhang L, Zhu P, Deng M, Huang C et al. Mammalian drug efflux transporters of the ATP binding cassette (ABC) family in multidrug resistance: a review of the past decade. Cancer Lett. (2016) 370:153-64. doi: 10.1016/j.canlet.2015.10.010

64. Zhao L, Liu W, Xiao J, Cao B. The role of exosomes and 'exosomal shuttle microRNA' in tumorigenesis and drug resistance. Cancer Lett. (2015) 356:33946. doi: 10.1016/j.canlet.2014.10.027

65. Torreggiani E, Roncuzzi L, Perut F, Zini N, Baldini N. Multimodal transfer of MDR by exosomes in human osteosarcoma. Int J Oncol. (2016) 49:189-96. doi: 10.3892/ijo.2016.3509

66. Mc Namee N, O'Driscoll L. Extracellular vesicles and anticancer drug resistance Biochim Biophys Acta. (2018) 1870:123-36. doi: 10.1016/j.bbcan.2018.07.003

67. Nawaz M, Fatima F, Nazarenko I, Ekström K, Murtaza I, Anees M, et al. Extracellular vesicles in ovarian cancer: applications to tumor biology, immunotherapy and biomarker discovery. Expert Rev Proteomics. (2016) 13:395-409. doi: 10.1586/14789450.2016.1165613

68. Maacha S, Bhat AA, Jimenez L, Raza A, Haris M, Uddin S, et al. Extracellular vesicles-mediated intercellular communication: roles in the tumor microenvironment and anti-cancer drug resistance. Mol Cancer. (2019) 18:55. doi: 10.1186/s12943-019-0965-7

69. Chen R, Wang G, Zheng Y, Hua Y, Cai Z. Drug resistance-related microRNAs in osteosarcoma: translating basic evidence into therapeutic strategies. J Cell Mol Med. (2019) 23:2280-92. doi: 10.1111/jcmm.14064
70. Li X, Seebacher NA, Hornicek FJ, Xiao T4, Duan Z. Application of liquid biopsy in bone and soft tissue sarcomas: Present and future. Cancer Lett. (2018) 439:66-77. doi: 10.1016/j.canlet.2018.09.012

71. Jeyaram A, Jay SM. Preservation and storage stability of extracellular vesicles for therapeutic applications. AAPS J. (2017) 20:1. doi: 10.1208/s12248-017-0160-y

72. Brady JV, Troyer RM, Ramsey SA, Leeper H, Yang L, Maier CS, et al. Preliminary proteomic investigation of circulating exosomes and discovery of biomarkers associated with the progression of osteosarcoma in a clinical model of spontaneous disease. Transl Oncol. (2018) 11:1137-46. doi: 10.1016/j.tranon.2018.07.004

73. Xu JF, Wang YP, Zhang SJ, Chen Y, Gu HF, Dou XF, et al. Exosomes containing differential expression of microRNA and mRNA in osteosarcoma that can predict response to chemotherapy. Oncotarget. (2017) 8:75968-78. doi: 10.18632/oncotarget.18373

74. Bao Q, Gong L, Wang J, Wen J, Shen Y, Zhang W. Extracellular vesicle RNA sequencing reveals dramatic transcriptomic alterations between metastatic and primary osteosarcoma in a liquid biopsy approach. Ann Surg Oncol. (2018) 25:2642-51. doi: 10.1245/s10434-018-6642-Z

75. Ayers L, Pink R, Carter DRF, Nieuwland R. Clinical requirements for extracellular vesicle assays. J Extracell Vesicles. (2019) 8:1593755. doi: 10.1080/20013078.2019.1593755

76. Van Deun J, Mestdagh P, Agostinis P, Akay Ö, Anand S, Anckaert J, et al. EVTRACK: transparent reporting and centralizing knowledge in extracellular vesicle research. Nat Methods. (2017) 14:228-32. doi: 10.1038/nmeth. 4185

77. Rayyan M, Zheutlin A, Byrd JB. Clinical research using extracellular vesicles: insights from the International Society for Extracellular Vesicles 2018 Annual Meeting. J Extracell Vesicles. (2018) 7:1535744. doi: 10.1080/20013078.2018.1535744

78. Iliescu FS, Vrtačnik D, Neuzil P, Iliescu C. Microfluidic technology for clinical applications of exosomes. Micromachines. (2019) 10:392. doi: $10.3390 / \mathrm{mil} 0060392$

79. Lu J, Pang J, Chen Y, Dong Q, Sheng J, Luo Y, et al. Application of microfluidic chips in separation and analysis of extracellular vesicles in liquid biopsy for cancer. Micromachines. (2019) 10:390. doi: 10.3390/milo 060390

Conflict of Interest: The authors declare that the research was conducted in the absence of any commercial or financial relationships that could be construed as a potential conflict of interest.

Copyright (c) 2019 Perut, Roncuzzi and Baldini. This is an open-access article distributed under the terms of the Creative Commons Attribution License (CC BY). The use, distribution or reproduction in other forums is permitted, provided the original author(s) and the copyright owner(s) are credited and that the original publication in this journal is cited, in accordance with accepted academic practice. No use, distribution or reproduction is permitted which does not comply with these terms. 